\title{
Pacific
}

Journal of

Mathematics

\section{DROPLET CONDENSATION AND ISOPERIMETRIC TOWERS}

Matteo Novaga, Andrei Sobolevski And Eugene Stepanov 


\title{
DROPLET CONDENSATION AND ISOPERIMETRIC TOWERS
}

\author{
Matteo Novaga, Andrei Sobolevski and Eugene Stepanov
}

\begin{abstract}
We consider a variational problem in a planar convex domain, motivated by the statistical mechanics of crystal growth in a saturated solution. The minimizers are constructed explicitly and are completely characterized.
\end{abstract}

\section{Introduction}

In understanding the physical phenomenon of droplet condensation or crystal growth, the central issue is to explain how a particular macroscopic shape of the growing droplet or crystal is determined by microscopic interactions of its constituent particles.

According to Gibbs' formulation of statistical mechanics, the probability of a microscopic configuration $\sigma$ is proportional to $\exp (-\beta H(\sigma))$, where $\beta>0$ is the inverse temperature and $H(\cdot)$ is the Hamiltonian defining the energy of the system. Therefore the most probable configurations are the ones with minimal energy. In the "thermodynamical" limit of a large number of particles, this minimum becomes very sharp: the overall configuration of the system settles, up to minute fluctuations, to a well-defined deterministic structure.

It turns out that the microscopic laws of atomic interactions give rise to a certain macroscopic quantity, the surface tension, which determines the droplet shape via minimization of the surface energy. Phenomenology of surface tension was proposed by Gibbs in the late 1870s. In an important contribution, G. Wulff suggested in 1900 that for a growing crystal, its equilibrium shape is that of a ball in a metric generated by the surface tension (the Wulff shape).

The first author acknowledges partial support by the Fondazione CaRiPaRo Project "Nonlinear Partial Differential Equations: models, analysis, and control-theoretic problems." The second author was partially supported by Laboratory for Structural Methods of Data Analysis in Predictive Modeling, MIPT, RF government grant, ag. 11.G34.31.0073, and by RFBR grant 11-01-93106 CNRSL_a. The work of the third author was financed by GNAMPA, by RFBR grant 11-01-00825, and by the project 2008K7Z249 "Trasporto ottimo di massa, disuguaglianze geometriche e funzionali e applicazioni" financed by the Italian Ministry of Research.

MSC2010: 28A75, 49Q20, 82B24.

Keywords: constrained isoperimetric problems, crystal growth, anisotropic variational problems. 
It has been furthermore observed experimentally that flat facets of a growing crystal may carry macroscopic but monomolecular "islands", whose shape is also determined by the surface tension. A mathematical approach to explaining this phenomenon has been developed by S. Shlosman and collaborators in a series of works [Schonmann and Shlosman 1996a; Ioffe and Shlosman 2008; 2010], building upon his earlier work with R. L. Dobrushin and R. Kotecky [Dobrushin et al. 1992].

A typical setting in this approach is represented by the following discrete model of crystal growth, which is a variant of the Ising model: fix an open domain $\Omega \subset \mathbb{R}^{2}$ of unit area and consider the three-dimensional lattice obtained by intersecting the cylinder $\Omega \times[-1,1] \subset \mathbb{R}^{3}$ with $(1 / N)\left(\mathbb{Z}^{3}+\left(0,0, \frac{1}{2}\right)\right)$, where $N$ is a large integer parameter. At each node $t$ of this lattice there is a variable $\sigma_{t}$ (the spin) taking values +1 (interpreted as " $t$ belongs to the free phase") and -1 (interpreted as " $t$ belongs to the condensed phase"). The collection $\sigma=\left(\sigma_{t}\right)$ is called the microscopic configuration of the system.

Fix now the Ising Hamiltonian $H(\sigma)=-\sum_{s, t:|s-t|=1} \sigma_{s} \sigma_{t}$, which describes a "ferromagnetic" interaction between nearest neighbors (equal values have smaller energy than opposite ones), and consider the canonical probability distribution $p(\sigma)=$ $\exp (-\beta H(\sigma)) / Z$. Here the normalization coefficient $Z=\sum_{\sigma} \exp (-\beta H(\sigma))$ is defined by summation over all configurations that satisfy the so-called Dobrushin boundary condition: spins at outermost nodes $(x, y, z)$ of the lattice have values +1 if $z>0$ and -1 if $z<0$.

It turns out that in the limit of large $N$ the main contribution to probability comes from configurations where the lower and upper halves of the lattice are filled, respectively, with -1 's and +1 's. In this equilibrium state, the numbers of +1 's and -1 's are asymptotically equal, so that $S_{N}=\sum_{t} \sigma_{t} \sim 0$, and fluctuations of the flat surface dividing the two phases are logarithmic in $N$.

A more interesting situation occurs when, in addition to the Dobrushin boundary values, the system is conditioned to have macroscopically more -1 's than +1 's:

$$
S_{N}=\sum_{t} \sigma_{t}=-m N^{2}
$$

with $m>0$. In this case, depending on the value of $m$, the most probable state of the system may feature one or more monomolecular layers on top of the surface $z=0$ in the box $\Omega \times[-1,1]$. A detailed account of the observed equilibrium states as $m$ changes can be found in [Ioffe and Shlosman 2010].

As proved in [Schonmann and Shlosman 1996a], the behavior of this model in the continuous limit $N \rightarrow \infty$ is closely related to the following variational problem: given an open set $\Omega \subset \mathbb{R}^{n}$ and a value $m \in[0,+\infty)$, find

(1-1) $\min \left\{\int_{\Omega} \varphi^{*}(D u): u \in B V\left(\mathbb{R}^{n}\right), u=0\right.$ on $\left.\mathbb{R}^{n} \backslash \Omega, u(\cdot) \in \mathbb{N}, \int_{\Omega} u d x=m\right\}$, 
where $\varphi^{*}$ is some given general norm on $\mathbb{R}^{n}$. Of course, in the application to the Ising model we are discussing here one has $n=2$, the two-dimensional case; however the case of generic dimension $n$ of the ambient space $\mathbb{R}^{n}$ also makes sense from the mathematical point of view. The growth of a droplet and formation of new layers of the solid is described by the growth of profile $u$ as $m$ increases.

The norm $\varphi^{*}(\cdot)$ here is related to the surface tension as follows. The surface tension $\gamma^{3 D}(\cdot)$ is a function defined over $\mathbb{S}^{2}$, the two-dimensional unit sphere in $\mathbb{R}^{3}$, and satisfying $\gamma^{3 D}(v) \geq 0$ and $\gamma^{3 D}(-v)=\gamma^{3 D}(v)$ for all $v \in \mathbb{S}^{2}$. The surface energy of a closed surface $M^{2} \subset \mathbb{R}^{3}$ is defined to be

$$
H\left(M^{2}\right)=\int_{M^{2}} \gamma^{3 D}\left(v_{s}\right) d s,
$$

where $v_{s}$ is the unit normal to $M^{2}$ at $s \in M^{2}$. While $\gamma^{3 D}$ defines the $3 D$ shape of a crystal growing in space, the shape of monolayers growing on facets is given by the restricted $2 D$ surface tension defined for $n \in \mathbb{S}^{1}$ by

$$
\gamma^{2 D}(v)=\left.\frac{\partial}{\partial v} \gamma^{3 D}\right|_{v_{s}=(0,0,1)},
$$

where the derivatives are taken at the "north pole" $v_{s}=(0,0,1) \in \mathbb{S}^{2}$ along all tangents $v \in \mathbb{S}^{1}$ to $\mathbb{S}^{2}$ [Ioffe and Shlosman 2010]. The function $\gamma^{2 D}$ can then be extended to all of $\mathbb{R}^{2}$ by homogeneity of degree one, and $\varphi^{*}(\cdot)$ is defined as the convex hull of the thus defined $\gamma^{2 D}(\cdot)$. However in the sequel $\varphi^{*}$ will be fixed, without any assumptions of smoothness or strict convexity: indeed one of the examples in Section 5 corresponds to a crystalline norm.

It is easy to see that the functional minimized in (1-1) is the one-dimensional surface energy for the restricted surface tension. It turns out that minimization of this surface energy alone is sufficient to reconstruct most of the physics of monomolecular layer growth described in [Ioffe and Shlosman 2010]. In particular, if $\varphi^{*}(\cdot)$ is the Euclidean norm and $\Omega$ a unit square, then as $m$ grows, the first four monomolecular layers start as Wulff circles and then develop into "Wulff plaquettes" while from the fifth layer on all new layers appear as Wulff plaquettes identical to underlying layers (Section 5).

In contrast, this simple variational model does not capture the thermodynamic fluctuations, which render Wulff circles below a certain size unstable and prevent their formation for small $m$. Neither does it capture the microscopic (that is, "finite$N$ ") structure of the Wulff plaquettes, whose boundaries are in fact separated with gaps that vanish in the continuous limit. A first-principle approach that takes proper account of these phenomena is due to R. Dobrushin, S. Shlosman and their coauthors and is presented in [Dobrushin et al. 1992; Schonmann and Shlosman 1996a; 1996b; Ioffe and Shlosman 2008; 2010]. 
It is worth observing that a similar problem with the additional restriction that $u$ be a characteristic function of some set (that is, that the droplet has exactly one layer) in the two-dimensional situation (that is when $n=2$ ), the set $\Omega$ is convex, and the norm $\varphi^{*}$ is Euclidean, has been studied in [Stredulinsky and Ziemer 1997], and for more general anisotropic norms (but for a somewhat different functional, namely, with penalization on the volume instead of the volume constraint), in [Novaga and Paolini 2005]. The latter problem will play an important role also in the present paper. Eventually, one has to mention that it is also very similar to the well-known Cheeger problem, the solutions of the latter being so-called Cheeger sets; see for instance [Buttazzo et al. 2007; Kawohl and Novaga 2008; Kawohl and Lachand-Robert 2006; Caselles et al. 2010].

Our aim in this paper is to study the variational problem (1-1) in the twodimensional case (that is, when $n=2$ ). This geometric optimization problem is considered without resort to the underlying lattice model or its continuous limit, allowing us to treat an arbitrary open domain $\Omega$ and an arbitrary norm $\varphi^{*}$ that is not necessarily strictly convex. In this setting we completely characterize the minimizers and the possible levels of $u$ when the domain $\Omega$ is convex. In particular it turns out that except some degenerate situation, which can however happen only when $\Omega$ is not strictly convex, the number of nonzero levels of $u$ is at most two.

The basic tool we use is the auxiliary problem when $u$ is a priori required to have a single nonzero level (that is, is requested to be a characteristic function); namely, we show that in the two-dimensional case $(n=2)$ when $\Omega$ is convex, the nonzero levels of solutions to the latter problem corresponding to different values of $m$ as $m$ grows can be arranged as a family of sets ordered by inclusion. Thus, solutions to problem (1-1) can be seen as "towers" with levels solving the auxiliary problem. The assumption of convexity of $\Omega$ is essential, as shown by a counterexample at the end of Section 4A. The main result of the paper is formulated as Theorem 4.10. We conclude with an explicit example of solutions to (1-1) for the case of a square $\Omega=[0,1]^{2}$ with a strictly convex (Euclidean) norm and a crystalline norm.

This work was inspired by some seminar talks of Senya Shlosman. After it was completed, we learned that a full description of the solutions to the variational problem (1-1) when $\Omega$ is a square and $\varphi^{*}$ is generated by a physical Hamiltonian (in particular, when it is the Euclidean norm) has been independently obtained by him and Ioffe by a rigorous continuous limit of a suitable lattice model (S. Shlosman, private communication, 2012). Their proof, together with an analysis of the microscopic structure of the solution and its behavior under thermal perturbations, has not yet been published. 


\section{Notation and preliminary results}

For a set $E \subset \mathbb{R}^{n}$ we denote by $|E|$ its Lebesgue measure, by $\mathbf{1}_{E}$ its characteristic function, by $\bar{E}$ its closure, by $\partial E$ its topological boundary, and by $E^{c}$ its complement.

In the following $\varphi$ will denote the given (not necessarily Euclidean) norm over $\mathbb{R}^{n}$. Given $E \subset \mathbb{R}^{n}$ and $x \in \mathbb{R}^{n}$, we set

$$
\operatorname{dist}_{\varphi}(x, E):=\inf _{y \in E} \varphi(x-y), \quad d_{\varphi}^{E}(x):=\operatorname{dist}_{\varphi}(x, E)-\operatorname{dist}_{\varphi}\left(x, E^{c}\right) .
$$

The value $d_{\varphi}^{E}(x)$ is the signed distance from $x$ to $\partial E$ and is positive outside $E$. Notice that at each point where $d_{\varphi}^{E}$ is differentiable one has (see [Bellettini et al. 2001])

$$
\varphi^{*}\left(\nabla d_{\varphi}^{E}\right)=1, \quad v \cdot \nabla d_{\varphi}^{E}=1 \quad \text { for all } v \in \partial \varphi^{*}\left(\nabla d_{\varphi}^{E}\right),
$$

where $\varphi^{*}$ denotes the dual norm of $\varphi$ defined as

$$
\varphi^{*}(\xi):=\max _{\eta: \varphi(\eta) \leq 1} \xi \cdot \eta
$$

and $\partial \varphi^{*}$ denotes the subdifferential of $\varphi^{*}$ in the sense of convex analysis. In particular

$$
\nabla d_{\varphi}^{E}=\frac{v^{E}}{\varphi^{*}\left(v^{E}\right)}
$$

where $v^{E}$ is the exterior Euclidean unit normal to $\partial E$.

We define the anisotropic perimeter of a set $E \subseteq \mathbb{R}^{n}$ as

$$
P_{\varphi}(E):=\sup \left\{\int_{E} \operatorname{div} \eta d x: \eta \in C_{0}^{1}\left(\mathbb{R}^{n}\right), \varphi(\eta) \leq 1\right\}=\int_{\partial^{*} E} \varphi^{*}\left(v^{E}\right) d \mathscr{H}^{n-1}
$$

where $\partial^{*} E$ is the reduced boundary of $E$ according to De Giorgi. We will usually identify a set $E$ of finite perimeter with the set of its density points (that is, points of density 1).

Given an open set $\Omega \subset \mathbb{R}^{n}$ we define the $B V$-seminorm of $v \in B V(\Omega)$ as

$$
\int_{\Omega} \varphi^{*}(D v):=\sup \left\{\int_{\Omega} v \operatorname{div} \eta d x: \eta \in C_{0}^{1}\left(\mathbb{R}^{n}\right), \varphi(\eta) \leq 1\right\},
$$

where $C_{0}^{1}\left(\mathbb{R}^{n}\right)$ stands for the set of continuously differentiable functions with compact support is $\mathbb{R}^{n}$.

We let $W_{\varphi}:=\{x \mid \varphi(x)<1\}$, usually called the Wulff shape, be the unit ball of $\varphi$. Observe that $P_{\varphi}\left(W_{\varphi}\right)=n\left|W_{\varphi}\right|$.

In the sequel, given $x \in \mathbb{R}^{n}$ and $r>0$, we set $W_{r}(x):=x+r W_{\varphi}$ (a Wulff ball of radius $r$ with center $x$ ). In this notation the reference to a norm $\varphi$ is not retained 
for the sake of brevity, but always silently assumed. When $\varphi$ is the Euclidean norm, we will use a more common notation $B_{r}(x)$ instead of $W_{r}(x)$ and $P$ instead of $P_{\varphi}$. Definition 2.1. Given an $r>0$, we say that $E$ satisfies the $r W_{\varphi}$-condition, if for every $x \in \partial E$ there exists an $y \in \mathbb{R}^{n}$ such that $W_{r}(y) \subset E$ and $x \in \partial W_{r}(y)$.

Observe that, if $E$ is convex, then $E^{c}$ satisfies the $r W_{\varphi}$-condition for all $r>0$.

We conclude the section by recalling the following isoperimetric inequality [Taylor 1975].

Proposition 2.2. For all $E \subset \mathbb{R}^{n}$ such that $|E|<+\infty$ there holds

$$
P_{\varphi}(E) \geq \frac{|E|^{\frac{n-1}{n}}}{\left|W_{\varphi}\right|^{\frac{n-1}{n}}} P_{\varphi}\left(W_{\varphi}\right) .
$$

\section{Existence of minimizers}

Notice that, since the total variation is lower semicontinuous and the constraints are closed under weak $B V$ convergence, by direct method one immediately gets existence of minimizers of (1-1).

Proposition 3.1. For any $m \geq 0$ there exists a (possibly nonunique) minimizer of (1-1).

For every $u \in L^{1}\left(\mathbb{R}^{n}\right)$ and $j \in \mathbb{N}$ we set

$$
E_{j}:=\{u \geq j\} .
$$

It is worth observing that whenever $u(\cdot)$ takes values in $\mathbb{N}$,

$$
u=\sum_{i=1}^{\infty} \mathbf{1}_{E_{i}}
$$

and

$$
\int_{\mathbb{R}^{n}} \varphi^{*}(D u)=\sum_{i=1}^{\infty} P_{\varphi}\left(E_{i}\right) .
$$

Remark 3.2. If we let $u_{m}$ be a minimizer of (1-1) for a given $m>0$, then the normalized functions $v_{m}:=u_{m} / m$ converge, as $m \rightarrow \infty$, up to a subsequence, to a minimizer of the problem

$$
\min \left\{\int_{\Omega} \varphi^{*}(D v): v \in B V\left(\mathbb{R}^{n}\right), v=0 \text { on } \Omega^{c}, \int_{\Omega} v d x=1\right\},
$$

which is closely related to the Cheeger problem in $\Omega$ [Kawohl and Novaga 2008]. Proposition 3.3. If $u$ is a minimizer of (1-1), then $u \in L^{\infty}\left(\mathbb{R}^{n}\right)$. 
Proof. Assume by contradiction that $\left|E_{j}\right|>0$ for all $j \in \mathbb{N}$. Notice that

$$
\lim _{j \rightarrow \infty}\left|E_{j}\right|=0
$$

(since otherwise $u$ would not be integrable). Given $x_{0} \in \Omega$ we let

$$
u_{j}:=\min (u, j)+\mathbf{1}_{W_{R_{j}}\left(x_{0}\right)}
$$

where the radius $R_{j}$ is such that

$$
\int_{\Omega} u_{j}=\int_{\Omega} u=m
$$

that is (keeping in mind (3-2)),

$$
\left|W_{\varphi}\right| R_{j}^{n}=\sum_{i>j}\left|E_{i}\right|
$$

and choose $j \in \mathbb{N}$ big enough so that $W_{R_{j}}\left(x_{0}\right) \subset \Omega$.

Letting

$$
f(t):=n\left|W_{\varphi}\right|^{\frac{1}{n}} t^{\frac{n-1}{n}} \quad \text { so that } P_{\varphi}\left(W_{R_{j}}\left(x_{0}\right)\right)=f\left(\left|W_{\varphi}\right| R_{j}^{n}\right),
$$

we have

$$
\begin{array}{rlrl}
\int_{\Omega} \varphi^{*}\left(D u_{j}\right) & \leq \int_{\Omega} \varphi^{*}(D \min (u, j))+P_{\varphi}\left(W_{R_{j}}\left(x_{0}\right)\right) \\
& =\int_{\Omega} \varphi^{*}(D \min (u, j))+f\left(\left|W_{\varphi}\right| R_{j}^{n}\right) & \\
& \leq \int_{\Omega} \varphi^{*}(D \min (u, j))+\sum_{i>j} f\left(\left|E_{i}\right|\right) \quad & \text { by the concavity of } f \\
& \leq \int_{\Omega} \varphi^{*}(D \min (u, j))+\sum_{i>j} P_{\varphi}\left(E_{i}\right) & & \text { by }(2-3) \\
& =\int_{\Omega} \varphi^{*}(D u) &
\end{array}
$$

the second inequality being strict unless $\left|E_{i}\right|=\left|E_{k}\right|$ for all $i>j, k>j$, thus leading to a contradiction.

Proposition 3.4. Let $\Omega \subset \mathbb{R}^{n}$ be star-shaped. Then the problem (1-1) is equivalent to the following relaxed problem:

(3-4) $\min \left\{\int_{\Omega} \varphi^{*}(D u): u \in B V\left(\mathbb{R}^{n}\right), u=0\right.$ on $\left.\Omega^{c}, u(\cdot) \in \mathbb{N}, \int_{\Omega} u d x \geq m\right\}$.

Namely, the minimum values and the minimizers are the same for both problems. 
Proof. It is enough to show that any minimizer $u$ of (3-4) satisfies

$$
\int_{\Omega} u d x=m
$$

To this aim let $\Omega$ be star-shaped with respect to $x_{0}$ and assume by contradiction that (3-5) is violated. Let $u_{\lambda}(x):=u\left(x_{0}+\lambda\left(x-x_{0}\right)\right)$ for any $\lambda>0$, so that $u_{\lambda} \in B V\left(\mathbb{R}^{n}\right)$, $u_{\lambda}(\cdot) \in \mathbb{N}$, while, by star-shapedness of $\Omega$, one has $u_{\lambda}=0$ outside of $\Omega$ for every $\lambda \geq 1$. Then there exists a $\lambda>1$ such that (3-5) holds with $u$ replaced by $u_{\lambda}$. However,

$$
\int_{\Omega} \varphi^{*}\left(D u_{\lambda}\right)=\lambda^{1-n} \int_{x_{0}+\lambda\left(\Omega-x_{0}\right)} \varphi^{*}(D u)=\lambda^{1-n} \int_{\Omega} \varphi^{*}(D u)<\int_{\Omega} \varphi^{*}(D u)
$$

(the second equality is due to the fact that $\Omega \subset x_{0}+\lambda\left(\Omega-x_{0}\right)$ for $\lambda>1$, while $u=0$ outside of $\Omega$ ), contradicting the minimality of $u$.

\section{The convex two-dimensional case}

In this section we shall assume that $n=2$ and $\Omega \subset \mathbb{R}^{2}$ is a convex open set.

Given $E \subset \mathbb{R}^{2}$ and an $r>0$ we define the set $E^{r} \subset E$ by the formula

$$
E^{r}:= \begin{cases}\bigcup\left\{W_{r}(x): W_{r}(x) \subset E\right\} & \text { if } r>0, \\ E & \text { if } r=0 .\end{cases}
$$

Notice that, if $E$ is a convex set, then $E^{r}$ is convex and satisfies the $r W_{\varphi}$-condition. The set $E^{r}$ is called the Wulff plaquette of radius $r$ relative to $E$.

The following assertion holds:

Lemma 4.1. Let $E \subset \mathbb{R}^{2}$ be a convex open set satisfying the $r W_{\varphi}$-condition for some $r>0$. Then $E=E^{r}$.

Proof. One has $E^{r} \subset E$. On the other hand, $\partial E \subset \partial E^{r}$ because $E$ satisfies the $r W_{\varphi}$-condition. Minding that $E$, and hence $E^{r}$, is convex, we get $E=E^{r}$.

The convexity of set $E$ is essential in Lemma 4.1. In fact, if $A, B$ and $C$ are the vertices of an equilateral triangle $\triangle A B C$ with side length 1 , then letting

$$
E:=B_{1 / 2}(A) \cup B_{1 / 2}(B) \cup B_{1 / 2}(C) \cup \triangle A B C
$$

we have that $E$ satisfies the $\frac{1}{2} W_{\varphi}$-condition with respect to the Euclidean norm, but

$$
E^{1 / 2}=B_{1 / 2}(A) \cup B_{1 / 2}(B) \cup B_{1 / 2}(C) \neq E .
$$


4A. Isoperimetric sets. We consider the constrained isoperimetric problem

$$
\min \left\{P_{\varphi}(E): E \subset \Omega,|E|=m \in[0,|\Omega|]\right\},
$$

which corresponds to the problem (1-1) under the additional constraint that $u$ is a characteristic function. Clearly, the minimizers of this problem exist and the assertion of Proposition 3.4 remains valid for this problem.

Let $R_{\Omega}>0$ be the maximal radius $R$ such that $W_{R}(x) \subseteq \Omega$ for some $x \in \Omega$, and let $r_{\Omega} \in\left[0, R_{\Omega}\right]$ be the maximal radius $r$ such that $\Omega$ satisfies the $r W_{\varphi}$-condition (we set for convenience $r_{\Omega}=0$ if $\Omega$ does not satisfy any $r W_{\varphi}$-condition). Observe that in the Euclidean case one has

$$
r_{\Omega}=\frac{1}{\|\kappa\|_{L^{\infty}(\partial \Omega)}}
$$

where $\kappa$ stands for the curvature of $\partial \Omega$.

Lemma 4.2. Let $m \in(0,|\Omega|)$, and let $E$ be a minimizer of (4-2). Then $E$ is convex and there exists an $r>0$ (depending on $m$ ) such that $E$ satisfies the $r W_{\varphi}$-condition and each connected component of $\partial E \cap \Omega$ is contained in $\partial W_{r}(x)$, for some Wulff ball $W_{r}(x) \subset \Omega$ (with $x$ depending on the connected component of $\partial E \cap \Omega$ ).

Remark 4.3. Recall that here and in the sequel when speaking of the properties of a set $E$ of finite perimeter we actually refer to the respective properties of the set of its density points. In particular, a minimizer $E$ of (4-2) is not necessarily convex, but the set of its density points is (and hence, in particular, the closure $\bar{E}$ is convex).

Proof. STEP 1. We first show the convexity of E. As in [Ambrosio et al. 2001, Theorem 2] we can uniquely decompose $E$ as a union of (measure theoretic) connected components $\left\{E_{i}\right\}_{i \in I}$, where $I$ is finite or countable, such that

$$
|E|=\sum_{i \in I}\left|E_{i}\right| \quad \text { and } \quad P_{\varphi}(E)=\sum_{i \in I} P_{\varphi}\left(E_{i}\right) .
$$

As in [Ambrosio et al. 2002, Proposition 6.12], one shows by the isoperimetric inequality and the minimality of $E$ that the number of connected components is finite and the boundary of each connected component $E_{i}$ is parametrized by a finite number of pairwise disjoint Jordan curves. In particular, the boundaries of two different connected components do not intersect. Further, using Lemma 6.9 from [Ambrosio et al. 2002], one has that the perimeter $P_{\varphi}\left(E_{i}\right)$ of a measure theoretic connected component $E_{i}$ that has its boundary parametrized by Jordan curves $\left\{\theta_{i}^{j}\right\}_{j=1}^{N_{i}}$ (all parametrized, say, over $[0,1]$ ) is given by

$$
P_{\varphi}\left(E_{i}\right)=\sum_{j=1}^{N_{i}} \int_{0}^{1} \psi\left(\dot{\theta}_{i}^{j}(t)\right) d t,
$$


where $\psi: \mathbb{R}^{2} \rightarrow \mathbb{R}$ is some convex and 1-homogeneous function (in fact, $\psi:=\varphi^{*} \circ R$, $R$ being the clockwise rotation of $\mathbb{R}^{2}$ by $\pi / 2$; see Corollary 6.10 from [Ambrosio et al. 2002]). Hence, using Jensen inequality one shows that the convex envelope of $E_{i}$ has lower (anisotropic) perimeter than $E_{i}$ itself, and minding that is also has greater volume (as well as the fact that the assertion of Proposition 3.4 is valid for the problem (4-2)), one has that each $E_{i}$ is convex.

Finally, if $E$ is not connected, recalling that $\Omega$ is convex we can translate a connected component inside $\Omega$ in such a way that its boundary touches the boundary of another connected component (this changes neither the perimeter nor the volume), and taking the convex envelope of the resulting set we obtain again a set with greater volume and strictly lower anisotropic perimeter, hence a contradiction which shows that $E$ is convex.

STEP 2. Reasoning as in [Novaga and Paolini 2005, Theorem 4.5], where the related problem

$$
\min \left\{P_{\varphi}(E)-\lambda|E|: E \subset \Omega, \lambda \geq 0\right\}
$$

was considered instead of (4-2), one gets that each connected component of $\partial E \cap \Omega$ is contained in $\partial W_{r}(x)$, for some $x \in \mathbb{R}^{2}$ and $r>0$.

Moreover, as in [Ambrosio et al. 2002, Theorem 6.19] one can show the existence of a (possibly nonunique) Lipschitz continuous vector field $n: \partial E \rightarrow \mathbb{R}^{2}$ such that $n(x) \in \partial \varphi^{*}(v(x))$ for $\mathscr{H}^{1}$-a.e. $x \in \partial E$. In particular $\operatorname{div}_{\tau} n \in L^{\infty}(\partial E)$, where $\operatorname{div}_{\tau} n:=\partial_{\tau}(n \cdot \tau)$ denotes the tangential divergence of $n$ and corresponds to the anisotropic curvature of $\partial E$; see [Taylor 1975; Bellettini et al. 2001]. (Here and below $\tau$ and $v$ denote respectively the Euclidean unit tangent and exterior normal vectors to $\partial E$.)

Without loss of generality we may assume that $\operatorname{div}_{\tau} n$ is constant along every maximal segment contained in $\partial E$ (if not, we can substitute $n$ over the segment by a convex combination of its values on the endpoints of the segment; one would then still have $n \in \partial \varphi^{*}(v)$ along the segment because $v$ is constant there and $\partial \varphi^{*}(\cdot)$ is convex). In particular, if a connected component $\Sigma$ of $\partial E \cap \Omega$ is contained in $\partial W_{r}(x)$, then $n(y)=(y-x) /(r \varphi(y-x))$ for $\mathscr{H}^{1}$-a.e. $y \in \Sigma$.

STEP 3. We now prove that $E$ satisfies the $r W_{\varphi}$-condition for some $r>0$. Since $E$ is convex, it is enough to show that

$$
\operatorname{div}_{\tau} n \leq \frac{1}{r} \quad \mathscr{H}^{1} \text {-a.e. on } \partial E .
$$

This follows by a local variation argument as in the proof of Lemma 4.9 below. Let us fix $x_{1} \in \Sigma$, where $\Sigma$ is a connected component of $\partial E \cap \Omega$, and $x_{2} \in \partial E \backslash \bar{\Sigma}$. We know from the previous step that $\Sigma$ is contained in $\partial W_{r}(x)$ for some $x \in \mathbb{R}^{2}$ and $r>0$. We distinguish four cases. 
Case 1. There are two disjoint open sets $U_{i}, i=1,2$, such that $x_{i} \in U_{i}$ and $U_{i} \cap \partial E$ do not contain segments. Let $\psi_{1}, \psi_{2}$ be two nonnegative smooth functions, with support on $U_{1}, U_{2}$ respectively, such that

$$
\int_{U_{1} \cap \partial E} \psi_{1}(z) \varphi^{*}(v(z)) d \mathscr{H}^{1}(z)=\int_{U_{2} \cap \partial E} \psi_{2}(z) \varphi^{*}(v(z)) d \mathscr{H}^{1}(z) .
$$

We consider a family of diffeomorphisms such that

$$
\Psi(\varepsilon, x):=x+\varepsilon \psi_{1}(x) n(x)-\varepsilon \psi_{2}(x) n(x)+o(\varepsilon)
$$

for $\varepsilon>0$ small enough. By (4-4), the term $o(\varepsilon)$ can be chosen in such a way that

$$
\left|E^{\varepsilon}\right|=|E| \text { for all } \varepsilon>0 \text { small enough, }
$$

with $E^{\varepsilon}:=\Psi(\varepsilon, E) \subset \Omega$. We then have

$$
\begin{aligned}
P_{\varphi}\left(E^{\varepsilon}\right)=P_{\varphi}(E)+\frac{\varepsilon}{r} \int_{U_{1} \cap \partial E} \psi_{1}(z) \varphi^{*}(v(z)) d \mathscr{H}^{1}(z) & \\
& -\varepsilon \int_{U_{2} \cap \partial E} \psi_{2}(z) \operatorname{div}_{\tau} n(z) \varphi^{*}(v(z)) d \mathscr{H}^{1}(z)+o(\varepsilon),
\end{aligned}
$$

where $v$ stands for the exterior Euclidean unit normal to $\partial E$. As $\varepsilon \rightarrow 0^{+}$, by minimality of $E$, we get

$$
\frac{1}{r} \int_{U_{1} \cap \partial E} \psi_{1}(z) \varphi^{*}(v(z)) d \mathscr{H}^{1}(z) \geq \int_{U_{2} \cap \partial E} \psi_{2}(z) \operatorname{div}_{\tau} n(z) \varphi^{*}(v(z)) d \mathscr{H}^{1}(z),
$$

which in view of (4-4) gives (4-3).

Case 2. We can find two maximal segments $\ell_{1}, \ell_{2} \subset \partial E$ such that $x_{i} \in \ell_{i}$, and we define $E^{\varepsilon}$ by shifting $\ell_{1}$ by $c_{1} \varepsilon$ parallel to itself outside $E$, and by shifting $\ell_{2}$ by $c_{2} \varepsilon$ inside of $E$, with $c_{1}, c_{2}$ so that (4-5) holds, that is

$$
c_{1}\left|\ell_{1}\right|=c_{2}\left|\ell_{2}\right| .
$$

By [Novaga and Paolini 2005, Lemma 4.4] we have

$$
P_{\varphi}\left(E^{\varepsilon}\right)=P_{\varphi}(E)+c_{1} \alpha_{1} \varepsilon-c_{2} \alpha_{2} \varepsilon+o(\varepsilon),
$$

where $\alpha_{1}, \alpha_{2}$ are respectively the (Euclidean) length of the face of $W_{\varphi}$ parallel to $\ell_{1}, \ell_{2}$. By minimality of $E$, letting $\varepsilon \rightarrow 0^{+}$we obtain $c_{1} \alpha_{1} \geq c_{2} \alpha_{2}$. Recalling (4-6), we finally get

$$
\frac{1}{r}=\frac{\alpha_{1}}{\left|\ell_{1}\right|} \geq \frac{\alpha_{2}}{\left|\ell_{2}\right|}=\operatorname{div}_{\tau} n(z) \quad \text { for } z \in \ell_{2}
$$


Case 3. There is a maximal segment $\ell_{1} \subset \partial E$ and an open set $U_{2}$ such that $x_{1} \in \ell_{1}$, $x_{2} \in U_{2}$ and $U_{2} \cap \partial E$ does not contain segments. We proceed by combining the previous strategies and we define the set $E^{\varepsilon}$ by shifting $\ell_{1}$ by $\varepsilon$ parallel to itself outside $E$, and then taking the image of the resulting set through the diffeomorphism

$$
\Psi(\varepsilon, x):=x-\varepsilon \psi_{2}(x) n(x)+o(\varepsilon),
$$

where $\psi_{2}$ is a nonnegative smooth function supported on $U_{2}$ satisfying

$$
\int_{U_{2} \cap \partial E} \psi_{2}(z) \varphi^{*}(v(z)) d \mathscr{H}^{1}(z)=\left|\ell_{1}\right| .
$$

This condition guarantees that the volume change after these two operations is of order $o(\varepsilon)$, so that the extra term $o(\varepsilon)$ in the definition of $\Psi$ is chosen in such a way that (4-5) holds. Reasoning as above, we get

$$
P_{\varphi}\left(E^{\varepsilon}\right)=P_{\varphi}(E)+\alpha_{1} \varepsilon-\varepsilon \int_{U_{2} \cap \partial E} \psi_{2}(z) \operatorname{div}_{\tau} n(z) \varphi^{*}(v(z)) d \mathscr{H}^{1}(z)+o(\varepsilon),
$$

which gives, by minimality of $E$,

$$
\alpha_{1}=\frac{\left|\ell_{1}\right|}{r} \geq \int_{U_{2} \cap \partial E} \psi_{2}(z) \operatorname{div}_{\tau} n(z) \varphi^{*}(v(z)) d \mathscr{H}^{1}(z),
$$

which gives (4-3), recalling (4-7).

Case 4. There is a maximal segment $\ell_{2} \subset \partial E$ and an open set $U_{1}$ such that $x_{1} \in U_{1}$, $x_{2} \in \ell_{2}$ and $U_{1} \cap \partial E$ does not contain segments. This case can be dealt with reasoning as in the previous case, by shifting $\ell_{2}$ by $\varepsilon$ inside $E$ and defining

$$
\Psi(\varepsilon, x):=x+\varepsilon \psi_{1}(x) n_{2}(x)+o(\varepsilon) .
$$

STEP 4. From (4-3) it follows that the radius $r$ in Step 3 does not depend on the connected component $\Sigma$. In particular, every connected component of $\partial E \cap \Omega$ is contained in $\partial W_{r}(x)$, for a fixed $r>0$ (while $x$ depends in general on the connected component).

Consider now the function $v(r):=\left|\Omega^{r}\right|$. It is clearly constantly equal to $|\Omega|$ for $r \leq r_{\Omega}$ and to zero for $r>R_{\Omega}$, while over $\left[r_{\Omega}, R_{\Omega}\right]$ it is continuous and monotone decreasing. In particular, for all $m \in\left[\left|\Omega^{R_{\Omega}}\right|,|\Omega|\right]$ there exists a unique value $r_{m} \in\left[r_{\Omega}, R_{\Omega}\right]$ such that $v\left(r_{m}\right)=m$.

From the isoperimetric inequality (2-3) and Lemma 4.2, we get the following statement.

Proposition 4.4. Let $\Omega \subset \mathbb{R}^{2}$ be convex, and let $E$ be a minimizer of (4-2) with $m \in[0,|\Omega|]$. Then either

(a) $\bar{E}=\bar{\Omega}^{r_{m}}$, if $m>\left|\Omega^{R_{\Omega}}\right|$, or 
(b) $\bar{E}$ is the closure of some convex union of Wulff balls of radius $R_{\Omega}$, if $m \in$ $\left[R_{\Omega}^{2}\left|W_{\varphi}\right|,\left|\Omega^{R_{\Omega}}\right|\right]$, or

(c) $\bar{E}=\bar{W} \sqrt{m /\left|W_{\varphi}\right|}(x)$ for some $x \in \Omega$, if $m \leq R_{\Omega}^{2}\left|W_{\varphi}\right|$.

Proof. We can assume $m \in(0,|\Omega|)$. By Lemma 4.2, there exists an $r>0$ (depending on $m$ ) such that $\bar{E}$ is the closure of a union of Wulff balls of radius $r$, hence $\bar{E} \subset \bar{\Omega}^{r}$ and $r \leq R_{\Omega}$.

If $m>\left|\Omega^{R_{\Omega}}\right|$, then necessarily $r<R_{\Omega}$ and $\bar{E}=\bar{\Omega}^{r}$, since otherwise we could find a connected component of $\partial E \cap \Omega$ that is not contained in the boundary of a Wulff ball, contradicting Lemma 4.2. In particular, we have $r=r_{m}$.

If $m \in\left[R_{\Omega}^{2}\left|W_{\varphi}\right|,\left|\Omega^{R_{\Omega}}\right|\right]$ then $r=R_{\Omega}$, since otherwise $\bar{E}$ would coincide with the set $\bar{\Omega}^{r}$ (with $r<R_{\Omega}$ ), which has volume strictly greater than $\left|\Omega^{R_{\Omega}}\right|$.

If $m \leq R_{\Omega}^{2}\left|W_{\varphi}\right|$ the result follows by the isoperimetric inequality (2-3).

Remark 4.5. It is worth noticing that, if $\Omega$ is strictly convex, then there exists a unique ball $W_{R_{\Omega}}(x) \subset \Omega$, and thus $\Omega^{R_{\Omega}}=W_{R_{\Omega}}(x)$. In other words, the case (b) of the above Proposition 4.4 reduces to case (c). Therefore, either $\bar{E}=\bar{\Omega}^{r_{m}}$, if $m \geq \mid \Omega^{R_{\Omega}}$, or $\bar{E}=\bar{W}_{\sqrt{m /\left|W_{\varphi}\right|}}(x)$ for some $x \in \Omega$, if $m \leq\left|\Omega^{R_{\Omega}}\right|$.

We now state an easy consequence of Proposition 4.4 showing that solutions to the problem (4-2) with decreasing volumes may be arranged as a decreasing sequence of sets.

Corollary 4.6. Let $\Omega$ be convex and let $m_{j}$ be a decreasing sequence such that $m_{j} \in(0,|\Omega|)$, for all $j$. There exists a sequence of sets $E_{j}$ such that $E_{j+1} \subset E_{j} \subset \Omega$, $\left|E_{j}\right|=m_{j}$ and each $E_{j}$ is a minimizer of (4-2) with $m:=m_{j}$.

Note that the convexity assumption on the set $\Omega$ is essential in the above result. In fact, reasoning as in [Kawohl and Lachand-Robert 2006, Section 6] with the example of $\Omega$ a couple of circles connected by a thin tube (like a barbell considered in [Kawohl and Lachand-Robert 2006, Section 6]), one provides a family of minimizers of (4-2) with decreasing volumes which cannot be arranged as a decreasing sequence of sets (see Figure 1).

4B. Isoperimetric towers. We return now to the original problem (1-1). Here and below we let $u \in L^{1}\left(\mathbb{R}^{2}\right)$ be an arbitrary minimizer of this problem and $E_{j}$ be its level set corresponding to a $j \in \mathbb{N}$, as defined by (3-1). The following result follows directly from Corollary 4.6.

Proposition 4.7. If $\Omega$ is convex, then for all $j \in \mathbb{N}$ the set $E_{j}$ is a minimizer of the problem (4-2) with $m:=\left|E_{j}\right|$ (in particular $E_{j}$ is convex).

Proof. If the assertion is not true, then considering a sequence of sets $E_{j}^{\prime}$ of minimizers of (4-2) (with $m:=\left|E_{j}\right|$ ) such that $E_{j+1}^{\prime} \subset E_{j}^{\prime} \subset \Omega,\left|E_{j}^{\prime}\right|:=\left|E_{j}\right|$ (the 


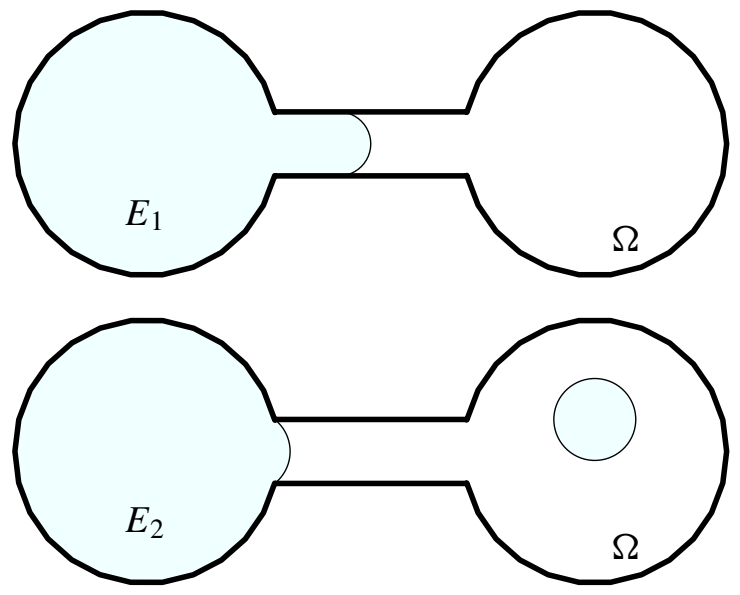

Figure 1. Example of $\Omega \subset \mathbb{R}^{2}$ nonconvex: two circles connected with a thin tube. $E_{1}$ and $E_{2}$ (which has two connected components) are two minimizers of (4-2) that are not included into one another.

existence of such a sequence is guaranteed by Corollary 4.6), and setting

$$
u^{\prime}:=\sum_{j} \mathbf{1}_{E_{j}^{\prime}}
$$

we get

$$
\int_{\mathbb{R}^{2}} \varphi^{*}\left(D u^{\prime}\right)=\sum_{j} P_{\varphi}\left(E_{j}^{\prime}\right)<\sum_{j} P_{\varphi}\left(E_{j}\right)=\int_{\mathbb{R}^{2}} \varphi^{*}(D u),
$$

the strict inequality being due to the fact that one of $E_{j}$ is not a minimizer of (4-2) (with $m:=\left|E_{j}\right|$ ) by assumption. On the other hand,

$$
\int_{\Omega} u^{\prime} d x=\int_{\Omega} u d x=m
$$

since the level sets of $u^{\prime}$ and $u$ have the same volume by construction. This would mean that $u$ is not a solution to the problem (1-1).

Remark 4.8. By Proposition 4.7 and Lemma 4.2, each set $E_{i}$ is convex and each connected component of $\partial E_{i} \cap \Omega$ is contained in $\partial W_{r_{i}}\left(x_{i}\right)$ for some Wulff ball $W_{r_{i}}\left(x_{i}\right) \subset \Omega$.

Lemma 4.9. Let $S_{i}, S_{j}$ be connected components of $\partial E_{i} \cap \Omega$ and $\partial E_{j} \cap \Omega$, respectively, with $j>i$, such that

$$
S_{i} \subset \partial W_{r_{i}}\left(x_{i}\right) \subset \bar{\Omega}, \quad S_{j} \subset \partial W_{r_{j}}\left(x_{j}\right) \subset \bar{\Omega}, \quad \frac{1}{r_{i}}\left(S_{i}-x_{i}\right) \subset \frac{1}{r_{j}}\left(S_{j}-x_{j}\right),
$$

for some $x_{i}, x_{j} \in \mathbb{R}^{2}, r_{i}, r_{j}>0$. Then $r_{i} \geq r_{j}$. 
Proof. It is enough to consider the case $j=i+1$. We can also assume $S_{i} \neq S_{i+1}$, otherwise there is nothing to prove. As in Figure 2, there are two cases to consider. Case 1. There are two points $y_{i} \in S_{i}, y_{i+1} \in S_{i+1}$ and two disjoint open sets $U_{i} \subset \Omega$ and $U_{i+1} \subset \Omega$ such that $y_{i} \in U_{i}, y_{i+1} \in U_{i+1}$, and that $U_{i} \cap S_{i}$ and $U_{i+1} \cap S_{i+1}$ do not contain segments. Consider a smooth function $\psi_{i}$ with support on $U_{i}$. It generates a one-parameter family of diffeomorphisms of $E_{i}$ defined by

$$
\Psi_{i}(\varepsilon, x):=x-\varepsilon \psi_{i}(x) n_{i}(x)
$$

for all sufficiently small $\varepsilon>0$, where

$$
n_{i}(x):=\frac{x-x_{i}}{r_{i} \varphi\left(x-x_{i}\right)} .
$$

Consider now a one-parameter family $\left\{\Psi_{i+1}(\varepsilon, \cdot)\right\}$ of diffeomorphisms of $E_{i+1}$ such that $\Psi_{i+1}(0, x)=x$ for all $x \in E_{i+1}, \Psi_{i+1}(\varepsilon, \cdot)-$ Id is supported in $U_{i+1}$ for all $\varepsilon>0$, while

$$
\Psi_{i+1}(\varepsilon, x):=x+\varepsilon \psi_{i+1}(x) n_{i+1}(x)+o(\varepsilon)
$$

as $\varepsilon \rightarrow 0^{+}$, where $\psi_{i+1}$ is some smooth function (with support in $U_{i+1}$ ), and

$$
n_{i+1}(x):=\frac{x-x_{i+1}}{r_{i+1} \varphi\left(x-x_{i+1}\right)} .
$$

We choose $\Psi_{i+1}$ so that the sets $E_{i}^{\varepsilon}:=\Psi_{i}\left(\varepsilon, E_{i}\right)$ and $E_{i+1}^{\varepsilon}:=\Psi_{i+1}\left(\varepsilon, E_{i+1}\right)$ satisfy

$$
\left|E_{i}^{\varepsilon}\right|+\left|E_{i+1}^{\varepsilon}\right|=\left|E_{i}\right|+\left|E_{i+1}\right|
$$

for all sufficiently small $\varepsilon>0$. Denote by $v_{j}$ the exterior Euclidean unit normal to $\partial E_{j}$. Since

$$
\begin{aligned}
\left|E_{i}^{\varepsilon}\right| & =\left|E_{i}\right|-\varepsilon \int_{\partial E_{i} \cap U_{i}} \psi_{i}(z) \varphi^{*}\left(\nu_{i}(z)\right) d \mathscr{H}^{1}(z)+o(\varepsilon), \\
\left|E_{i+1}^{\varepsilon}\right| & =\left|E_{i+1}\right|+\varepsilon \int_{\partial E_{i+1} \cap U_{i+1}} \psi_{i+1}(z) \varphi^{*}\left(\nu_{i+1}(z)\right) d \mathcal{H}^{1}(z)+o(\varepsilon),
\end{aligned}
$$

as $\varepsilon \rightarrow 0^{+}$, we have

$$
\int_{\partial E_{i} \cap U_{i}} \psi_{i}(z) \varphi^{*}\left(v_{i}(z)\right) d \mathscr{H}^{1}(z)=\int_{\partial E_{i+1} \cap U_{i+1}} \psi_{i+1}(z) \varphi^{*}\left(v_{i+1}(z)\right) d \mathscr{H}^{1}(z) .
$$

Letting now

$$
u_{\varepsilon}:=u-\mathbf{1}_{E_{i}}-\mathbf{1}_{E_{i+1}}+\mathbf{1}_{E_{i}^{\varepsilon}}+\mathbf{1}_{E_{i+1}^{\varepsilon}}=\sum_{\substack{k \neq i \\ k \neq i+1}} \mathbf{1}_{E_{k}}+\mathbf{1}_{E_{i}^{\varepsilon}}+\mathbf{1}_{E_{i+1}^{\varepsilon}},
$$


we have $\int_{\Omega} u_{\varepsilon} d x=\int_{\Omega} u d x$ for all sufficiently small $\varepsilon>0$. Recall that

$$
\begin{aligned}
\int_{\Omega} \varphi^{*}\left(D u_{\varepsilon}\right)=\int_{\Omega} \varphi^{*}(D u) & -\varepsilon \int_{\partial E_{i} \cap U_{i}} \frac{1}{r_{i}} \psi_{i}(z) \varphi^{*}\left(v_{i}(z)\right) d \mathcal{H}^{1}(z) \\
& +\varepsilon \int_{\partial E_{i+1} \cap U_{i+1}} \frac{1}{r_{i+1}} \psi_{i+1}(z) \varphi^{*}\left(v_{i+1}(z)\right) d \mathcal{H}^{1}(z)+o(\varepsilon) .
\end{aligned}
$$

As $\varepsilon \rightarrow 0^{+}$, by minimality of $u$, we get

$$
-\frac{1}{r_{i}} \int_{\partial E_{i} \cap U_{i}} \psi_{i}(z) \varphi^{*}\left(v_{i}(z)\right) d \mathscr{H}^{1}(z)+\frac{1}{r_{i+1}} \int_{\partial E_{i+1} \cap U_{i+1}} \psi_{i+1}(z) \varphi^{*}\left(v_{i+1}(z)\right) d \mathscr{H}^{1}(z)
$$

which together with (4-9) implies the thesis.

Case 2. We can find two maximal line segments $\ell_{i} \subset S_{i}$ and $\ell_{i+1} \subset S_{i+1}$. We define then $E_{i}^{\varepsilon}$ by shifting the segment $\ell_{i}$ by $c_{i} \varepsilon$ parallel to itself inside $E_{i}$ and $E_{i+1}^{\varepsilon}$ by shifting the segment $\ell_{i+1}$ parallel to itself outside of $E_{i+1}$ by $c_{i+1} \varepsilon$ with $c_{i}$ and $c_{i+1}$ so as to satisfy

$$
\left|E_{i}^{\varepsilon}\right|+\left|E_{i+1}^{\varepsilon}\right|=\left|E_{i}\right|+\left|E_{i+1}\right|
$$

for all $\varepsilon>0$ sufficiently small. Since

$$
\begin{aligned}
\left|E_{i}^{\varepsilon}\right| & =\left|E_{i}\right|-c_{i}\left|\ell_{i}\right| \varepsilon+o(\varepsilon), \\
\left|E_{i+1}^{\varepsilon}\right| & =\left|E_{i+1}\right|+c_{i+1}\left|\ell_{i+1}\right| \varepsilon+o(\varepsilon),
\end{aligned}
$$

as $\varepsilon \rightarrow 0^{+}$, we have

$$
c_{i}\left|\ell_{i}\right|=c_{i+1}\left|\ell_{i+1}\right|
$$

Letting again, as in Case 1,

$$
u_{\varepsilon}:=u-\mathbf{1}_{E_{i}}-\mathbf{1}_{E_{i+1}}+\mathbf{1}_{E_{i}^{\varepsilon}}+\mathbf{1}_{E_{i+1}^{\varepsilon}}=\sum_{\substack{k \neq i \\ k \neq i+1}} \mathbf{1}_{E_{k}}+\mathbf{1}_{E_{i}^{\varepsilon}}+\mathbf{1}_{E_{i+1}^{\varepsilon}},
$$

we have $\int_{\Omega} u_{\varepsilon} d x=\int_{\Omega} u d x$ for all sufficiently small $\varepsilon>0$. On the other hand, by [Novaga and Paolini 2005, Lemma 4.4],

$$
\int_{\Omega} \varphi^{*}\left(D u_{\varepsilon}\right)=\int_{\Omega} \varphi^{*}(D u)-c_{i} \alpha_{i} \varepsilon+c_{i+1} \alpha_{i+1} \varepsilon+o(\varepsilon)
$$

where $\alpha_{i}, \alpha_{i+1}$ are the (Euclidean) lengths of the face of $W_{\varphi}$ parallel to $\ell_{i}, \ell_{i+1}$, respectively. By minimality of $u$, letting $\varepsilon \rightarrow 0^{+}$we obtain $c_{i} \alpha_{i} \leq c_{i+1} \alpha_{i+1}$. Recalling (4-10), we get $r_{i}=\left|\ell_{i}\right| / \alpha_{i} \geq\left|\ell_{i+1}\right| / \alpha_{i+1}=r_{i+1}$.

Notice that in this proof we do not have to deal with the situation depicted in Cases 3 and 4 of the proof of Lemma 4.2 due to condition (4-8). In fact, the latter implies that if $S_{i}$ contains a line segment $\ell_{i}$, then the line segment $\ell_{j}:=$ 
$x_{j}+\left(l_{i}-x_{i}\right) r_{j} / r_{i}$ is contained in $S_{j}$. Otherwise, if there is a neighborhood $U_{i}$ of a point of $S_{i}$ such that $S_{i} \cap U_{i}$ does not contain any line segment, then $U_{j}:=x_{j}+\left(U_{i}-x_{i}\right) r_{j} / r_{i}$ is a neighborhood of a point in $S_{j}$ such that $S_{j} \cap U_{j}$ does not contain any line segment.
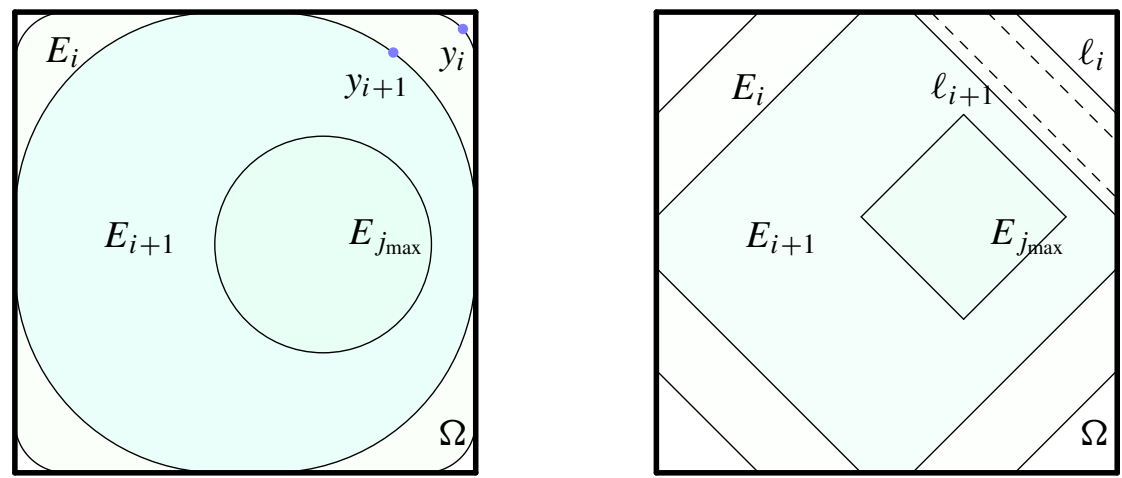

Figure 2. The two possible cases in the proof of Lemma 4.9.

We are now able to prove the following result giving the complete characterization of solutions to the problem (1-1).

Theorem 4.10. Let $\Omega \subset \mathbb{R}^{2}$ be convex and set $j_{\max }:=\|u\|_{\infty}$. Then one of the following cases holds.

(a) There exists an $\bar{r} \in\left[r_{\Omega}, R_{\Omega}\right)$ such that $\bar{E}_{j}=\bar{\Omega}^{\bar{r}}$ for all $j \leq j_{\max }$. In this case

$$
u=j_{\max } \mathbf{1}_{\Omega^{\bar{r}}}
$$

(in particular, if $\bar{r}=r_{\Omega}$, then $u=j_{\max } \mathbf{1}_{\Omega}$ ).

(b) There exists an $\bar{r} \in\left(r_{\Omega}, R_{\Omega}\right)$ such that $\bar{E}_{j_{\max }}=\bar{W}_{\bar{r}}(x)$ for some $x \in \Omega$ such that $W_{\bar{r}}(x) \subset \Omega^{\bar{r}}$, and $\bar{E}_{j}=\bar{\Omega}^{\bar{r}}$ for all $j<j_{\max }$. In this case

$$
u=\mathbf{1}_{W_{\bar{r}}(x)}+\left(j_{\max }-1\right) \mathbf{1}_{\Omega^{\bar{r}}} .
$$

(c) There exists an $\bar{r} \in\left(0, r_{\Omega}\right]$ such that $\bar{E}_{j_{\max }}=\bar{W}_{\bar{r}}(x)$ for some $x \in \Omega$ such that $W_{\bar{r}}(x) \subset \Omega$, and $\bar{E}_{j}=\bar{\Omega}$ for all $j<j_{\max }$. In this case

$$
u=\mathbf{1}_{W_{\bar{r}}(x)}+\left(j_{\max }-1\right) \mathbf{1}_{\Omega}
$$

(note that this condition may hold only when $r_{\Omega}>0$ ). 
(d) Every $\bar{E}_{j}$ is the closure of a convex union of Wulff balls of radius $R_{\Omega}$ for all $j \leq j_{\max }$. In this case

$$
u=\sum_{j=1}^{j_{\max }} \mathbf{1}_{E_{j}} .
$$

Remark 4.11. Observe that Case (d) of Theorem 4.10 is the only case where the number of nonzero level sets of the minimizer may be bigger than two.

Proof. We may assume $j_{\max }>1$, since otherwise the result follows directly from Proposition 4.4.

By Remark 4.8, for all $i \leq j_{\max }$ the set $E_{i}$ is convex and each connected component of $\partial E_{i} \cap \Omega$ is contained, up to a translation, in $\partial W_{r_{i}}\left(x_{i}\right)$ for some $r_{i}>0, x_{i} \in \mathbb{R}^{2}$. Moreover, if $\partial E_{i} \cap \Omega$ and $\partial E_{i+1} \cap \Omega$ are nonempty, from the inclusion $E_{i+1} \subset E_{i}$ it follows that we can always find two connected components $S_{i} \subset \partial E_{i} \cap \Omega$ and $S_{i+1} \subset \partial E_{i+1} \cap \Omega$ satisfying the assumptions of Lemma 4.9. By Lemma 4.9 we then get $r_{i} \geq r_{i+1}$ for all $i<j_{\max }$.

Recalling Propositions 4.7 and 4.4, this leaves only the following possibilities:

(i) $\bar{E}_{i}=\bar{\Omega}^{r_{i}}, \bar{E}_{i+1}=\bar{\Omega}^{r_{i+1}}$ with $r_{i} \geq r_{i+1}$. If $r_{i}>r_{\Omega}$ (hence $\Omega^{r_{i}} \neq \Omega$ ), then minding $\bar{E}_{i+1} \subset \bar{E}_{i}$ we have in this case $r_{i}=r_{i+1}$ hence $\bar{E}_{i}=\bar{E}_{i+1}=\bar{\Omega}^{r_{i}}$, while if $r_{i+1} \leq r_{i} \leq r_{\Omega}$ we have $\bar{E}_{i}=\bar{E}_{i+1}=\bar{\Omega}$, and we may just set $r_{i}=r_{i+1}:=r_{\Omega}$ so that still $\bar{E}_{i}=\bar{E}_{i+1}=\bar{\Omega}^{r_{i}}$ (because $\Omega^{r_{\Omega}}=\Omega$ ).

(ii) $\bar{E}_{i}=\bar{\Omega}^{r_{i}}, \bar{E}_{i+1}=\bar{W}_{r_{i+1}}\left(x_{i+1}\right)$ with $r_{i} \geq r_{i+1}$.

(iii) $\bar{E}_{i}=\bar{W}_{r_{i}}\left(x_{i}\right), \bar{E}_{i+1}=\bar{W}_{r_{i+1}}\left(x_{i+1}\right)$ with $r_{i} \geq r_{i+1}$.

(iv) $\bar{E}_{i}$ is a closure of some convex union of Wulff balls of radius $R_{\Omega}$ and $E_{i+1}=$ $\bar{W}_{r_{i+1}}\left(x_{i+1}\right)$ with $R_{\Omega}>r_{i+1}$.

(v) Both $\bar{E}_{i}$ and $\bar{E}_{i+1}$ are closures of some convex unions of Wulff balls of radius $R_{\Omega}$.

(Note that the case when $\bar{E}_{i+1}$ is a closure of a convex union of Wulff balls of radius $R_{\Omega}$ and $\bar{E}_{i}=\bar{W}_{r_{i}}\left(x_{i}\right)$ with $R_{\Omega}<r_{i}$ is impossible.) Thus there is a $\bar{j} \in\left\{0, \ldots, j_{\max }\right\}$ and an $r_{1} \in\left[r_{\Omega}, R_{\Omega}\right)$ such that either

(A) for every $i \leq \bar{J}$ one has $\bar{E}_{i}=\bar{\Omega}^{r_{1}}$ or

(B) for every $i \leq \bar{J}$ each $\bar{E}_{i}$ is a closure of a convex union of Wulff balls of radius $R_{\Omega}$ (in particular, just a single closed Wulff ball), while $\bar{E}_{i}=\bar{W}_{r_{i}}\left(x_{i}\right), r_{i}<R_{\Omega}$ for all $i>\bar{j}$, with $\left\{r_{i}\right\}$ decreasing.

Consider now an arbitrary $i>\bar{j}$ such that $E_{i} \neq \varnothing$. Note that either $r_{i} \leq r_{1}<R_{\Omega}$ (Case $\mathrm{A})$ or $r_{i}<R_{\Omega}$ (Case $\left.\mathrm{B}\right)$.

It remains to show that $E_{j}=\varnothing$ for all $j>i$. Suppose the contrary, namely, that $E_{i+1} \neq \varnothing$. We may assume without loss of generality all level sets are open convex 
by Proposition 4.7, and, further, $\bar{E}_{l+1} \subset E_{l}$ for all $l \in\left\{\bar{J}, \ldots, j_{\max }\right\}$ ) (if not, from what has been already proven it follows that we may just shift appropriately all the respective level sets, which would maintain both $\int_{\Omega} \varphi^{*}(D u)$ and $\left.\int_{\Omega} u\right)$. Choose now $\varepsilon>0$ and $\varepsilon^{\prime}>0$ sufficiently small so that for $r_{i+1}^{\prime}:=r_{i+1}-\varepsilon^{\prime}$ and $r_{i}:=r_{i}+\varepsilon$ one would have

$$
r_{i}^{\prime 2}+r_{i+1}^{\prime 2}=r_{i}^{2}+r_{i+1}^{2}
$$

and $\bar{W}_{r_{i}^{\prime}}\left(x_{i}\right) \subset E_{i-1}, \bar{E}_{i+2} \subset W_{r_{i+1}^{\prime}}\left(x_{i+1}\right)$. From (4-11) one gets $\varepsilon^{\prime}=\left(r_{i} / r_{i+1}\right) \varepsilon+$ $o(\varepsilon)$, and hence

$$
\frac{P_{\varphi}\left(W_{r_{i+1}^{\prime}}\left(x_{i+1}\right)\right)+P_{\varphi}\left(W_{r_{i}^{\prime}}\left(x_{i}\right)\right)}{P_{\varphi}\left(W_{r_{i+1}}\left(x_{i+1}\right)\right)+P_{\varphi}\left(W_{r_{i}}\left(x_{i}\right)\right)}=\frac{r_{i+1}^{\prime}+r_{i}^{\prime}}{r_{i+1}+r_{i}}=1-\varepsilon \frac{r_{i+1}-r_{i}}{r_{i+1}\left(r_{i+1}+r_{i}\right)}+o(\varepsilon),
$$

where the error term $o(\varepsilon)$ is negative when $r_{i}=r_{i+1}$. Therefore, representing $u$ as $u=\tilde{u}+\mathbf{1}_{E_{i+1}}+\mathbf{1}_{E_{i}}$, and letting

$$
u_{\varepsilon}^{\prime}:=\tilde{u}+\mathbf{1}_{W_{r_{i+1}^{\prime}}\left(x_{i+1}\right)}+\mathbf{1}_{W_{r_{i}^{\prime}}\left(x_{i}\right)},
$$

we get $\int_{\Omega} \varphi^{*}\left(D u_{\varepsilon}^{\prime}\right)<\int_{\Omega} \varphi^{*}(D u)$ for sufficiently small $\varepsilon>0$; but $\int_{\mathbb{R}^{2}} u d x=$ $\int_{\mathbb{R}^{2}} u_{\varepsilon}^{\prime} d x$, contrary to the optimality of $u$, which proves the claim.

One has therefore either $\bar{j}=j_{\max }-1$ or $\bar{j}=j_{\max }$, which concludes the proof.

\section{An explicit example}

5A. A square with Euclidean norm. Let now $\Omega:=[0,1]^{2}$ and let $\varphi$ be the Euclidean norm on $\mathbb{R}^{2}$. From Theorem 4.10 we obtain the following characterization for the minimizers of (1-1).

Proposition 5.1. Let $\Omega=[0,1]^{2}$.

(1) If $m \in(n-1, n \pi / 4)$, with $1 \leq n \leq 4$, we have $j_{\max }=n, \bar{E}_{j_{\max }}=\bar{B}_{r}\left(x_{0}\right) \subset \bar{\Omega}$ and $\bar{E}_{j}=\bar{\Omega}^{r}$ for $j<j_{\max }$, with

$$
r=\sqrt{\frac{n-m-1}{4(n-1)-n \pi}} .
$$

(2) If $m \in[n \pi / 4, n]$, with $1 \leq n \leq 4$, we have $j_{\max }=n$ and $\bar{E}_{j}=\bar{\Omega}^{r}$ for $j \leq j_{\max }$, with $r=\sqrt{(1-m / n) /(4-\pi)}$.

(3) If $m>4$ we have

$$
j_{\max } \in\left\{\left\lfloor\frac{2+\sqrt{\pi}}{2 \sqrt{\pi}} m\right\rfloor,\left\lfloor\frac{2+\sqrt{\pi}}{2 \sqrt{\pi}} m\right\rfloor+1\right\}
$$

and $\bar{E}_{j}=\bar{\Omega}^{r}$ for $j \leq j_{\max }$, with $r=\sqrt{\left(1-m / j_{\max }\right) /(4-\pi)}$. 
Proof. By Theorem 4.10 for all $m>0$ we have one of the following two possibilities. Case A. $\bar{E}_{j}=\bar{\Omega}^{r}$ for all $j \leq j_{\max }$ with

$$
m=j_{\max }\left|\Omega^{r}\right|=j_{\max }\left(1-(4-\pi) r^{2}\right) \quad r \in\left[0, \frac{1}{2}\right] .
$$

It then follows that

$$
r=r_{A}\left(j_{\max }\right):=\sqrt{\frac{j_{\max }-m}{(4-\pi) j_{\max }}}
$$

and $\sum_{j=1}^{j_{\max }} P\left(E_{j}\right)=F_{A}\left(j_{\max }\right)$, where

$$
F_{A}(x):=x P\left(\Omega^{r_{A}(x)}\right)=4 x-2 \sqrt{4-\pi} \sqrt{x(x-m)} .
$$

Notice that

$$
F_{A}^{\prime}(x)=4-\sqrt{4-\pi} \frac{2 x-m}{\sqrt{x(x-m)}},
$$

which implies that $F_{A}(x)$ is increasing for $x>((2+\sqrt{\pi}) / 2 \sqrt{\pi}) m$, while it is decreasing for $m \leq x<((2+\sqrt{\pi}) / 2 \sqrt{\pi}) m$. As a consequence we have

$$
j_{\max } \in\left\{j^{A}, j^{A}+1\right\}, \quad \text { where } j^{A}:=\left\lfloor\frac{2+\sqrt{\pi}}{2 \sqrt{\pi}} m\right\rfloor .
$$

Case B. $\bar{E}_{j_{\max }}=\bar{B}_{r}\left(x_{0}\right) \subset \bar{\Omega}$ and $\bar{E}_{j}=\bar{\Omega}^{r}$ for all $j<j_{\max }$ with

$$
\begin{aligned}
m & =\pi r^{2}+\left(j_{\max }-1\right)\left|\Omega^{r}\right| \\
& =\left(j_{\max }-1\right)\left(1-\left(4-\frac{j_{\max }}{j_{\max }-1} \pi\right) r^{2}\right) \quad r \in\left(0, \frac{1}{2}\right) .
\end{aligned}
$$

It follows

$$
r_{B}\left(j_{\max }\right):=\sqrt{\frac{j_{\max }-1-m}{(4-\pi)\left(j_{\max }-1\right)-\pi}}>r_{A}\left(j_{\max }-1\right)
$$

and $\sum_{j=1}^{j_{\max }} P\left(E_{j}\right)=F_{B}\left(j_{\max }\right)$, where

$$
\begin{aligned}
F_{B}(x): & =(x-1) P\left(\Omega^{r_{B}(x)}\right)+2 \pi r_{B}(x) \\
& =4(x-1)-2 \sqrt{4-\pi} \sqrt{\left(x-1-\frac{\pi}{4-\pi}\right)(x-1-m)} .
\end{aligned}
$$

Notice that the derivative

$$
F_{B}^{\prime}(x)=4-\sqrt{4-\pi} \frac{2(x-1)-m-\frac{\pi}{4-\pi}}{\sqrt{\left(x-1-\frac{\pi}{4-\pi}\right)(x-1-m)}} .
$$


Assuming $x>4 /(4-\pi)$, we then have that $F_{B}$ is increasing for

$$
x-1>\frac{2+\sqrt{\pi}}{2 \sqrt{\pi}} m-\frac{\sqrt{\pi}}{2(2+\sqrt{\pi})}
$$

and decreasing otherwise, so that

$$
j_{\max } \in\left\{j^{B}, j^{B}+1\right\}, \quad \text { where } j^{B}:=\left\lfloor\frac{2+\sqrt{\pi}}{2 \sqrt{\pi}} m+\frac{4+\sqrt{\pi}}{2(2+\sqrt{\pi})}\right\rfloor
$$

as soon as $j_{\max } \geq 5$.

Observe that, if $m<5 \pi / 4$, we have $j_{\max } \leq 4$ and there is only one choice for the minimizers $E_{j}$. In particular, we are in Case A or Case B depending on the value of $m$. On the other hand, when $m>5 \pi / 4$, we have to determine which one between Cases A and B is energetically more convenient. However, since $\min \left\{F_{B}\left(j^{B}\right), F_{B}\left(j^{B}+1\right)\right\}>\min \left\{F_{A}\left(j^{A}\right), F_{A}\left(j^{A}+1\right)\right\}$ for all $m>5 \pi / 4$, it follows that Case $\mathrm{B}$ can never occur as a minimizer, thus implying the thesis.

It is worth remarking that $2 \pi /(2+\pi)$ is the volume of the (unique) Cheeger set $C_{\Omega}$ of $\Omega$, so that Proposition 5.1 implies that the functions $u_{m} / j_{\max }$ converge to the characteristic function of $C_{\Omega}$, according to the Remark 3.2.

5B. A square with a crystalline norm. Now we set $\Omega=[0,1]^{2}$ as above and $\varphi(v)=\max \left\{\left|v_{1}\right|,\left|v_{2}\right|\right\}$. Notice that $\varphi$ is a crystalline norm with Wulff shape

$$
W_{\varphi}=\left\{(x, y) \in \mathbb{R}^{2}:|x|+|y| \leq 1\right\} .
$$

As before, we are able to characterize completely the minimizers of (1-1).

Proposition 5.2. Let $\Omega$ and $\varphi$ be as above.

(i) If $m \in\left(0, \frac{1}{2}\right]$, we have $j_{\max }=1$ and $\bar{E}_{1}=\bar{W}_{r}\left(x_{0}\right) \subset \bar{\Omega}$, with $r=\sqrt{m / 2}$.

(ii) If $m \in\left[\frac{1}{2}, 1\right)$, we have $j_{\max }=1$ and $\bar{E}_{1}=\bar{\Omega}^{r}$, with $r=\sqrt{(1-m) / 2}$.

(iii) If $m=1$, then either $j_{\max }=1$ and $\bar{E}_{1}=\Omega$, or $j_{\max }=2, \bar{E}_{1}=\bar{\Omega}^{r}$ and $\bar{E}_{2}=\bar{W}_{r}\left(x_{0}\right) \subset \Omega$, with $r \in\left(0, \frac{1}{2}\right]$.

(iv) If $m>1$, we have

$$
j_{\max } \in\left\{\left\lfloor\frac{1+\sqrt{2}}{2} m\right\rfloor,\left\lfloor\frac{1+\sqrt{2}}{2} m\right\rfloor+1\right\}
$$

and $\bar{E}_{j}=\bar{\Omega}^{r}$ for $j \leq j_{\max }$, with $r=\sqrt{\left(1-m / j_{\max }\right) / 2}$.

Proof. The proof is similar to that of Proposition 5.1.

If $m \leq \frac{1}{2}$, then $j_{\max }=1$ and $\bar{E}_{1}=\bar{W}_{r}\left(x_{0}\right) \subset \Omega$, since the (rescaled) Wulff shape solves the isoperimetric problem. By Theorem 4.10, for all $m \geq \frac{1}{2}$ we have one of the following two possibilities. 
Case A. $\bar{E}_{j}=\bar{\Omega}^{r}$ for all $j \leq j_{\max }$ with

$$
m=j_{\max }\left|\Omega^{r}\right|=j_{\max }\left(1-2 r^{2}\right), \quad r \in\left[0, \frac{1}{2}\right],
$$

which gives

$$
r=\frac{1}{\sqrt{2}} \sqrt{1-\frac{m}{j_{\max }}}
$$

and $\sum_{j=1}^{j_{\max }} P_{\varphi}\left(E_{j}\right)=F_{A}\left(j_{\max }\right)$, where

$$
F_{A}(x)=x(4-4 r)=4 x-4 \sqrt{\frac{x^{2}-m x}{2}} .
$$

Since the function $F_{A}$ is increasing for $x>(1+\sqrt{2}) m / 2$ and decreasing for $m \leq x<(1+\sqrt{2}) m / 2$, we have

$$
j_{\max } \in\left\{j^{A}, j^{A}+1\right\}, \quad \text { where } j^{A}:=\left\lfloor\frac{1+\sqrt{2}}{2} m\right\rfloor .
$$

Case B. $\bar{E}_{j_{\max }}=\bar{W}_{r}\left(x_{0}\right) \subset \bar{\Omega}$ and $\bar{E}_{j}=\bar{\Omega}^{r}$ for all $j<j_{\max }$, with $r \in\left(0, \frac{1}{2}\right]$ and

$$
m=2 r^{2}+\left(j_{\max }-1\right)\left(1-2 r^{2}\right),
$$

and hence $m \geq 1$ because $j_{\max } \geq 2$ and $r \leq \frac{1}{2}$.

If $m=1$ then $j_{\max }=2$ and we can take any $r \in\left(0, \frac{1}{2}\right]$.

If $m>1$ then $j_{\max } \geq m+1$ and we get

$$
r=\sqrt{\frac{j_{\max }-m-1}{2\left(j_{\max }-2\right)}}
$$

and $\sum_{j=1}^{j_{\max }} P_{\varphi}\left(E_{j}\right)=F_{B}\left(j_{\max }\right)$, where

$$
F_{B}(x)=4(x-1)-4(x-2) \sqrt{\frac{x-m-1}{2(x-2)}} .
$$

Since the function $F_{B}$ is increasing for $x>(1+\sqrt{2}) m / 2+(3-\sqrt{2}) / 2$ and decreasing otherwise, we have

$$
j_{\max } \in\left\{j^{B}, j^{B}+1\right\}, \quad \text { where } j^{B}:=\left\lfloor\frac{1+\sqrt{2}}{2} m+\frac{3-\sqrt{2}}{2}\right\rfloor .
$$

As in the proof of Proposition 5.1, when $m>1$ we have to determine which of Cases A and B is energetically more convenient. Since $\min \left\{F_{B}\left(j^{B}\right), F_{B}\left(j^{B}+1\right)\right\}>$ $\min \left\{F_{A}\left(j^{A}\right), F_{A}\left(j^{A}+1\right)\right\}$ (by a calculation as in the example with the Euclidean norm), it follows that Case B can never occur. 


\section{References}

[Ambrosio et al. 2001] L. Ambrosio, V. Caselles, S. Masnou, and J.-M. Morel, "Connected components of sets of finite perimeter and applications to image processing", J. Eur. Math. Soc. (JEMS) 3:1 (2001), 39-92. MR 2002g:49072 Zbl 0981.49024

[Ambrosio et al. 2002] L. Ambrosio, M. Novaga, and E. Paolini, "Some regularity results for minimal crystals", ESAIM Control Optim. Calc. Var. 8 (2002), 69-103. MR 2003h:49062 Zbl 1066.49021

[Bellettini et al. 2001] G. Bellettini, M. Novaga, and M. Paolini, "On a crystalline variational problem, II: $B V$ regularity and structure of minimizers on facets”, Arch. Ration. Mech. Anal. 157:3 (2001), 193-217. MR 2002c:49072b Zbl 0976.58017

[Buttazzo et al. 2007] G. Buttazzo, G. Carlier, and M. Comte, "On the selection of maximal Cheeger sets”, Differential Integral Equations 20:9 (2007), 991-1004. MR 2008i:49025 Zbl 1212.49019

[Caselles et al. 2010] V. Caselles, A. Chambolle, and M. Novaga, "Some remarks on uniqueness and regularity of Cheeger sets”, Rend. Semin. Mat. Univ. Padova 123 (2010), 191-201. MR 2012d:52015 Zbl 1198.49042

[Dobrushin et al. 1992] R. Dobrushin, R. Kotecký, and S. Shlosman, Wulff construction: A global shape from local interaction, Translations of Mathematical Monographs 104, American Mathematical Society, Providence, RI, 1992. MR 93k:82002 Zbl 0917.60103

[Ioffe and Shlosman 2008] D. Ioffe and S. Shlosman, "Ising model fog drip: the first two droplets", pp. 365-381 in In and out of equilibrium. 2, edited by V. Sidoravicius and M. E. Vares, Progr. Probab. 60, Birkhäuser, Basel, 2008. MR 2010i:82032 Zbl 1173.82311

[Ioffe and Shlosman 2010] D. Ioffe and S. Shlosman, "Ising fog drip: The shallow puddle, $o(n)$ deep", pp. 31-36 in Excess self-intersections and related topics (Marseille, 2010), Actes des rencontres du CIRM 2:1, 2010.

[Kawohl and Lachand-Robert 2006] B. Kawohl and T. Lachand-Robert, "Characterization of Cheeger sets for convex subsets of the plane", Pacific J. Math. 225:1 (2006), 103-118. MR 2007e:52002 Zbl 1133.52002

[Kawohl and Novaga 2008] B. Kawohl and M. Novaga, "The $p$-Laplace eigenvalue problem as $p \rightarrow 1$ and Cheeger sets in a Finsler metric", J. Convex Anal. 15:3 (2008), 623-634. MR 2009c:35336 Zbl 1186.35115

[Novaga and Paolini 2005] M. Novaga and E. Paolini, "Regularity results for boundaries in $\mathbb{R}^{2}$ with prescribed anisotropic curvature", Ann. Mat. Pura Appl. (4) 184:2 (2005), 239-261. MR 2006h: 49062 Zbl 1158.49306

[Schonmann and Shlosman 1996a] R. H. Schonmann and S. B. Shlosman, "Constrained variational problem with applications to the Ising model", J. Statist. Phys. 83:5-6 (1996), 867-905. MR 97h:82030 Zbl 1081.82547

[Schonmann and Shlosman 1996b] R. H. Schonmann and S. B. Shlosman, "Constrained variational problem with applications to the Ising model”, J. Statist. Phys. 83:5-6 (1996), 867-905. MR 97h:82030 Zbl 1081.82547

[Stredulinsky and Ziemer 1997] E. Stredulinsky and W. P. Ziemer, "Area minimizing sets subject to a volume constraint in a convex set”, J. Geom. Anal. 7:4 (1997), 653-677. MR 99k:49089 Zbl 0940.49025

[Taylor 1975] J. E. Taylor, "Unique structure of solutions to a class of nonelliptic variational problems", pp. 419-427 in Differential geometry (Stanford, 1973), vol. 1, edited by S. S. Chern and R. Osserman, Proc. Sympos. Pure. Math. 27, Amer. Math. Soc., Providence, RI, 1975. MR 52 \#9062 Zbl 0317.49054 
Received March 1, 2012. Revised September 24, 2012.

MatTeo Novaga

DiPARTIMENTO DI MATEMATICA

UNIVERSITÀ DI PADOVA

VIA TRIESTE 63

I-35121 PADOVA

ITALY

novaga@math.unipd.it

ANDREI SOBOLEVSKI

INSTITUTE FOR INFORMATION TRANSMISSION PROBLEMS (KHARKEVICH INSTITUTE)

19 B. KARETNY PER.

127994 MosCOW

RUSSIA

and

NATiONAL RESEARCH University Higher SCHOOL OF ECONOMICS

20 MYASNITSKAYA ST.

101000 MosCOW

RUSSIA

sobolevski@iitp.ru

EUGENE STEPANOV

Department of Mathematical Physics, Faculty of Mathematics and Mechanics

St. Petersburg State University

UNIVERSITETSKIJ PR. 28, OLD PETERHOF

198504 St. PETERSBURG

RUSSIA

and

St. Petersburg Branch of the Steklov Mathematical Institute of the Russian ACADEMY OF SCIENCES

FONTANKA 27

191023 St. Petersburg

RUSSIA

stepanov.eugene@gmail.com 


\title{
PACIFIC JOURNAL OF MATHEMATICS
}

\author{
msp.org/pjm
}

Founded in 1951 by E. F. Beckenbach (1906-1982) and F. Wolf (1904-1989)

\section{EDITORS}

V. S. Varadarajan (Managing Editor)

Department of Mathematics

University of California

Los Angeles, CA 90095-1555

pacific@math.ucla.edu

Paul Balmer

Department of Mathematics

University of California

Los Angeles, CA 90095-1555

balmer@math.ucla.edu

Daryl Cooper

Department of Mathematics

University of California

Santa Barbara, CA 93106-3080 cooper@math.ucsb.edu

Jiang-Hua $\mathrm{Lu}$

Department of Mathematics

The University of Hong Kong

Pokfulam Rd., Hong Kong jhlu@maths.hku.hk
Don Blasius

Department of Mathematics University of California

Los Angeles, CA 90095-1555

blasius@math.ucla.edu

Robert Finn

Department of Mathematics Stanford University

Stanford, CA 94305-2125

finn@math.stanford.edu

Sorin Popa

Department of Mathematics

University of California

Los Angeles, CA 90095-1555

popa@math.ucla.edu

Paul Yang

Department of Mathematics

Princeton University

Princeton NJ 08544-1000

yang@math.princeton.edu

\section{PRODUCTION}

Silvio Levy, Scientific Editor, production@msp.org

\section{SUPPORTING INSTITUTIONS}

ACADEMIA SINICA, TAIPEI

CALIFORNIA INST. OF TECHNOLOGY

INST. DE MATEMÁTICA PURA E APLICADA

KEIO UNIVERSITY

MATH. SCIENCES RESEARCH INSTITUTE

NEW MEXICO STATE UNIV.

OREGON STATE UNIV.

\author{
STANFORD UNIVERSITY \\ UNIV. OF BRITISH COLUMBIA \\ UNIV. OF CALIFORNIA, BERKELEY \\ UNIV. OF CALIFORNIA, DAVIS \\ UNIV. OF CALIFORNIA, LOS ANGELES \\ UNIV. OF CALIFORNIA, RIVERSIDE \\ UNIV. OF CALIFORNIA, SAN DIEGO \\ UNIV. OF CALIF., SANTA BARBARA
}

\author{
Vyjayanthi Chari \\ Department of Mathematics \\ University of California \\ Riverside, CA 92521-0135 \\ chari@math.ucr.edu \\ Kefeng Liu \\ Department of Mathematics \\ University of California \\ Los Angeles, CA 90095-1555 \\ liu@math.ucla.edu \\ Jie Qing \\ Department of Mathematics \\ University of California \\ Santa Cruz, CA 95064 \\ qing@cats.ucsc.edu
}

These supporting institutions contribute to the cost of publication of this Journal, but they are not owners or publishers and have no responsibility for its contents or policies.

See inside back cover or msp.org/pjm for submission instructions.

The subscription price for 2013 is US \$400/year for the electronic version, and \$485/year for print and electronic.

Subscriptions, requests for back issues and changes of subscribers address should be sent to Pacific Journal of Mathematics, P.O. Box 4163, Berkeley, CA 94704-0163, U.S.A. The Pacific Journal of Mathematics is indexed by Mathematical Reviews, Zentralblatt MATH, PASCAL CNRS Index, Referativnyi Zhurnal, Current Mathematical Publications and the Science Citation Index.

The Pacific Journal of Mathematics (ISSN 0030-8730) at the University of California, c/o Department of Mathematics, 798 Evans Hall \#3840, Berkeley, CA 94720-3840, is published monthly except July and August. Periodical rate postage paid at Berkeley, CA 94704, and additional mailing offices. POSTMASTER: send address changes to Pacific Journal of Mathematics, P.O. Box 4163, Berkeley, CA 94704-0163.

PJM peer review and production are managed by EditFLOW ${ }^{\circledR}$ from Mathematical Sciences Publishers.

PUBLISHED BY

mathematical sciences publishers

nonprofit scientific publishing

http://msp.org/

(C) 2013 Mathematical Sciences Publishers 


\section{PACIFIC JOURNAL OF MATHEMATICS}

Volume $262 \quad$ No. $2 \quad$ April 2013

Certifying incompressibility of noninjective surfaces with scl

DANNY CALEGARI

Global well-posedness for the 3D rotating Navier-Stokes equations with highly oscillating initial data

QIONGLEI CHEN, CHANGXING MiAO and ZHIFEI ZHANG

Presenting Schur superalgebras

HOUSSEIN El TURKEY and JONATHAN R. KUJAWA

Classifying zeros of two-sided quaternionic polynomials and computing zeros of two-sided polynomials with complex coefficients

FENG LIANGGUI and ZHAO KAIMING

Coxeter groups, imaginary cones and dominance

XIANG FU

Semicontinuity of automorphism groups of strongly pseudoconvex domains: The low differentiability case

Robert E. Greene, Kang-Tae Kim, Steven G. Krantz and

AERYEONG SEO

Klein four-subgroups of Lie algebra automorphisms

JING-SONG HUANG and JUN YU

Fractal entropy of nonautonomous systems

Rui KuANG, WEN-ChiaO CHENG and BING LI

A GJMS construction for 2-tensors and the second variation of the total

$Q$-curvature

YoshiniKo MaTSUMOTO

Droplet condensation and isoperimetric towers

Matteo Novaga, Andrei Sobolevski and Eugene Stepanov

Brauer's height zero conjecture for metacyclic defect groups

BENJAMIN SAMBALE 